Revue d'histoire de l'enfance « irrégulière »

Le Temps de l'histoire

5 | 2003

Pratiques éducatives et systèmes judiciaires

\title{
La gestion de la déviance des filles à Montréal au XIXe siècle. Les institutions du Bon-Pasteur d'Angers (1869-1912)
}

Véronique Strimelle

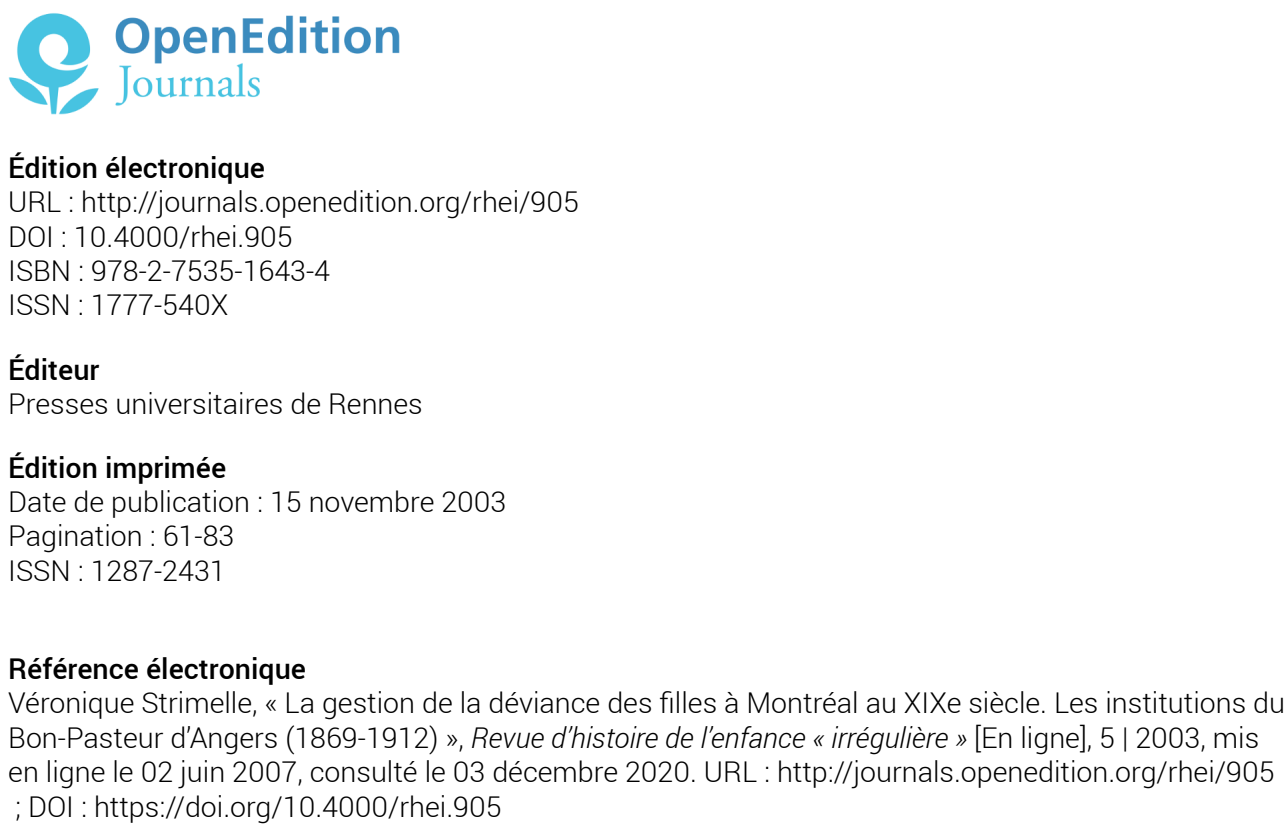




\section{La gestion de la déviance des filles à Montréal au XIXème siècle : les institutions du Bon-Pasteur d'Angers (1869-1912)}

Le 7 juin 1844, quatre religieuses du monastère du Bon-Pasteur d'Angers arrivent à Montréal. À la demande de l'évêque du lieu, Monseigneur Bourget, la supérieure de la communauté, Mère Marie-de-Sainte-Euphrasie, a délégué ce petit groupe de religieuses françaises afin de prendre en charge les femmes et les filles "repenties" de la région montréalaise. Vingt-cinq ans plus tard, en 1869, la communauté du Bon-Pasteur d'Angers est solidement implantée sur le territoire montréalais et a diversifié ses modes d'intervention. Un monastère imposant, bâti en 1847, accueille 349 personnes. ${ }^{(2)}$ Outre les 91 religieuses et 25 novices, l'établissement compte 84 élèves pensionnaires, 39 préservées, 91 pénitentes et 18 madeleines. ${ }^{(3)}$

L'année 1869 marque aussi une étape importante dans l'extension de l'œuvre des Sœurs du Bon-Pasteur auprès des filles et des femmes jugées déviantes. En effet, en avril, deux lois provinciales sont sanctionnées : l'Acte concernant les écoles d'industrie ${ }^{(4)}$ et l'Acte concernant les écoles de réforme, ${ }^{(5)}$ lois-cadres qui permettent l'édification au Québec d'un ensemble d'institutions destinées au traitement d'enfants délinquants, abandonnés, négligés ou maltraités. D’après ces deux lois, l'État a le pouvoir d'accorder le statut d'école de réforme ou d'école d'industrie "certifiée" à des institutions privées qui en font la demande et répondent aux exigences requises. ${ }^{(6)} \mathrm{D}$ 'après la loi, la population détenue doit aussi être répartie suivant la confession, chaque groupe religieux se devant alors d'organiser ses propres établissements. ${ }^{(7)}$

À Montréal, ce sont les Frères de la Charité de Saint-Vincent-de-Paul qui ouvrent une école de réforme pour garçons catholiques : l'institut Saint-Antoine. ${ }^{(8)}$ En ce qui concerne les filles, les Sœurs du Bon-Pasteur font leur demande d'accréditation en 1869. Dès 1870, elles peuvent ouvrir

\section{Véronique Strimelle ${ }^{(1)}$}

(1) Maître de conférences, université d'Ottawa.

(2) Anonyme, Annales des religieuses de Notre-

Dame-de-Charité du BonPasteur d'Angers à

Montréal, depuis leur

établissement jusqu'à 1896, Montréal, monastère du Bon-Pasteur, p. 50.

(3) Ibid., p. 50.

(4) Statuts du Québec, 32 Vict. (1869), ch. 17.

(5) Statuts du Québec, 32 Vict. (1869), ch. 18.

(6) Sur la genèse de cette loi et la mise en place des écoles de réforme et d'industrie, voir Jean-Marie Fecteau, Jean

Véronique Strimelle / p. 61 à 83 
Trépanier, Sylvie

Ménard et Véronique

Strimelle, «Une politi-

que de l'enfance délinquante et en danger :

la mise en place

des écoles de réforme et

d'industrie au Québec

(1840-1873) ", Crime,

bistoire \& sociétés, vol. 2, $\mathrm{n}^{\circ} 1,1998$, p. 75-110.

(7) Véronique

Strimelle, La gestion de la déviance des filles et les institutions du Bon-Pasteur à Montréal (1869-1912), thèse de doctorat en criminologie, université de Montréal, 1998, p. 91.

(8) Sylvie Ménard, L'institut Saint-Antoine et la problématique de réforme des garçons délinquants au Québec (1873-1909), thèse de doctorat en histoire, université du Québec à Montréal, 1998, 304 p.

(9) Véronique Strimelle, op. cit., p. 93, et Édouard Gouin, une école de réforme et une école d'industrie destinées aux jeunes catholiques dans leur vaste monastère de la rue Sherbrooke.(9) Ces institutions pour filles sont l'objet de notre étude, qui couvre la période 1869-1912, années où les modes de traitement de l'enfance délinquante et malheureuse subissent de profondes transformations. En histoire, comme en criminologie, il a fallu attendre les années 1980 pour que la question des filles et des femmes déviantes soit considérée comme un sujet de recherche per se. Le contrôle social des mineures au Canada anglais a fait l'objet d'un mémoire de maîtrise. ${ }^{(10)}$ Carolyn Strange a traité des femmes et des filles déviantes à Toronto. ${ }^{(1)}$ Pour Montréal, Tamara Myers s'est intéressée aux mécanismes de régulation et de traitement des mêmes populations au début du XXème siècle. Elle a notamment mis en évidence le rôle joué par la cour des jeunes délinquants en tant que mécanisme de contrôle et de prise en charge des mineures. ${ }^{(12)}$ Ces recherches, particulièrement celles d'inspiration féministe, ont permis d'appréhender ce nouveau champ d'intérêt selon un angle neuf. Cependant, la plupart de ces écrits interrogent plus les discours sur la délinquance des filles que les modalités concrètes de gestion de la déviance. Or, ces dernières peuvent faire ressortir un aspect très instructif de l'enfermement. L'intérêt porté aux pratiques d'enfermement permet en effet de vérifier dans quelle mesure les problèmes soulevés par la pratique ont pu affecter les cadres théoriques, idéologiques et légaux mis en place pour fixer l'existence des institutions, et de poser ainsi les questions de leur efficacité, ainsi que de leur réactivité aux conditions concrètes d'enfermement.

Dans le cadre des institutions que nous avons étudiées, la gestion des populations détenues revient presque exclusivement aux religieuses du Bon-Pasteur. Ainsi, dans le présent article, nous nous intéresserons tout spécialement à cette communauté et aux méthodes qu'elle entend appliquer aux filles dont elle a la charge. Après avoir situé la communauté du Bon-Pasteur et décrit brièvement les étapes de son histoire à Montréal, nous mettrons en évidence les valeurs et les méthodes éducatives développées par les religieuses dans le cadre de leur travail auprès des filles. Ayant dégagé certains traits caractéristiques de la vie au sein des institutions du Bon-Pasteur, nous pourrons ensuite comparer les modes d'en- 
fermement des filles et des garçons et nous interroger en finale sur la place accordée aux filles dans les politiques d'intervention pénale mises en œuvre au Québec à la fin du XIXème siècle.

\section{Les religieuses}

Comment des religieuses françaises appelées au Québec pour venir s'occuper des "filles perdues" furent-elles amenées à prendre en charge les jeunes délinquantes et les filles abandonnées dans le cadre des institutions créées par la loi de 1869 ? Pour répondre à cette question, nous nous interrogerons en premier lieu sur les origines et la vocation de cette communauté. En second lieu, nous aborderons l'histoire des institutions montréalaises gérées par la communauté entre 1844 et 1912.

\subsection{Les origines de la communauté}

Le premier fondateur de la communauté est saint Jean-Eudes (16011680), prêtre d'origine normande qui s'intéresse notamment au sort des femmes et des jeunes filles poussées à la prostitution et à d'autres comportements réprouvés par la société de l'époque. ${ }^{(13)}$ C'est pour s'occuper de ce type de population que Jean Eudes ouvre, en 1641, un refuge dans la ville de Caen. Une communauté de religieuses est alors fondée pour prendre en charge les "pénitentes" enfermées : l'institut du Refuge, qui prit ensuite le nom d'institut de Notre-Dame-de-Charité..$^{(14)}$

Huit refuges de Notre-Dame-de-Charité sont créés entre 1642 et 1724, notamment à Tours. À la même époque, il existe d'autres établissements qui recueillent ou gardent de force les "filles perdues", les enfants rebelles ou les ex-prisonnières. Ainsi, à la fin de l'Ancien Régime, on compte une vingtaine d'établissements de ce genre dans la ville de Paris. Parmi eux, une communauté de pénitentes, appelées par la suite Filles du BonPasteur, est fondée en 1688 par Marie de Combe. Cette communauté œuvre dans un établissement de la rue du Cherche-Midi, qui porte très vite le nom de "Bon-Pasteur". Ce terme devient au fil du temps synonyme d'institution d'enfermement pour la réforme morale des femmes et des filles. La dénomination de "Bon-Pasteur" n'est donc pas exclusive à une seule congrégation religieuse..$^{(15)}$
Le Bon-Pasteur et ses

auvres à Montréal,

Montréal, Institution des sourds-muets, 1916, p. 25.

(10) Bruno Théoret,

L'histoire du contrôle social et pénal des filles au Canada anglais de 1800 à 1930, mémoire de maîtrise en criminologie, université de Montréal, 1987, 138 p.

(11) Carolyn Strange, Toronto's girl problem, the perils and pleasures of the city, 1880-1930, Toronto, University of Toronto Press, 1995, 299 p.

(12) Tamara Myers, Criminal women and bad girls. Regulation and punishment in Montreal, 1890-1930, thèse de doctorat en histoire, université Mc Gill, 1995 , 318 p., et « The voluntary delinquent : Parents, daughters and the Montreal juvenile delin- 
quents' court in 1918 ", The Canadian Historical Review, vol. $80, \mathrm{n}^{\circ} 2$, juin 1999 , p. 242-268.

(13) Édouard Gouin, op. cit., p. 5.

(14) Ibid., p. 6.

(15) Christian Carlier, La prison aux champs. Les colonies d'enfants délinquants du Nord de la France au XIXème siècle, Paris, 1994, p. 240.

(16) Anonyme, Au soir d'un siècle. Le Bon-Pasteur d’Angers à Montréal, 1844-1944, Montréal, Monastère du BonPasteur, p. 31.

(17) Édouard Gouin, op. cit., p. 10.

(18) Anonyme, Au soir d'un siècle..., op. cit., p. 32 .

(19) Ibid, p. 32.
Après la Révolution de 1789, certains refuges de Notre-Dame-deCharité sont rouverts, mais les communautés recomposées rencontrent de graves problèmes matériels. En 1825, l'élection d'une nouvelle supérieure au refuge de Tours marque une étape décisive dans l'évolution de la communauté. Rose-Virginie Pelletier, en religion Mère Marie-deSainte-Euphrasie, accède au poste de supérieure à l'âge de vingt-huit ans. Dès son arrivée, elle n'a de cesse de développer de nouvelles fondations destinées aux femmes et aux jeunes filles "repenties". En 1831, Mère Marie-de-Sainte-Euphrasie est appelée à diriger le nouveau couvent d'Angers. Suivant les anciennes règles en usage dans les refuges de Notre-Dame-de-Charité, chaque nouvelle fondation garde son autonomie par rapport aux décisions de la maison-mère. Devenue supérieure, Mère Marie-de-Sainte-Euphrasie désire changer une telle situation car elle y voit un obstacle à l'établissement d'un réseau efficace et organisé de couvents. Pour ce faire, la supérieure entend instaurer un généralat, statut permettant de regrouper toutes les communautés isolées sous l'autorité d'une maison-mère. Tous les refuges fondés sous l'ancien système rejettent ce projet. Malgré une très vive opposition, Mère Mariede-Sainte-Euphrasie le soumet alors au Saint-Siège qui approuve l'instauration du généralat en $1835{ }^{\left({ }^{(1)}\right.} \mathrm{A}$ partir de ce moment, la communauté de Notre-Dame-de-Charité du Bon-Pasteur d'Angers forme une famille religieuse distincte des anciennes communautés, placée sous la direction d'une même supérieure et soumise à une même règle. ${ }^{(17)}$

Désireuse d'organiser la vie des couvents dans ses moindres détails et de former efficacement ses religieuses, Mère Marie-de-Sainte-Euphrasie développe, dans ses Entretiens, les principes et méthodes dont doivent s'inspirer les religieuses du Bon-Pasteur dans leur travail auprès des filles et des femmes. ${ }^{(18)}$ En plus de quarante ans de supériorat, elle réussit à faire du couvent d'Angers le centre d'une œuvre au rayonnement intense. À sa mort, en 1868, la communauté du Bon-Pasteur compte 900 religieuses et 110 monastères répartis dans de nombreux pays. En 1916, le nombre de monastères a plus que doublé : ils sont 260 et la communauté regroupe 10.028 religieuses..$^{(19)}$ 


\subsection{Les débuts à Montréal (1844-1869)}

Les religieuses du Bon-Pasteur arrivent à Montréal en 1844. Très rapidement, la communauté prend en charge les femmes "pénitentes" qui demandent asile ou celles qui sont envoyées par le tribunal. ${ }^{(20)}$ Dès 1847, les religieuses s'occupent aussi des "préservées", enfants de moins de douze ans qui n'ont pas commis de délits mais sont abandonnées, orphelines ou maltraitées. ${ }^{(21)}$ À ce groupe s'ajoutent les jeunes délinquantes destinées à la prison mais envoyées au Bon-Pasteur pour éviter le contact "pervertissant" des adultes. La communauté de Montréal accueille enfin une section de madeleines, communauté religieuse composée d'anciennes pénitentes ou de femmes "repenties". ${ }^{(22)}$

Tout en diversifiant assez rapidement leur œuvre, les religieuses du Bon-Pasteur de Montréal ne peuvent accueillir, par manque de place, qu'un nombre limité de filles. La construction d'un monastère sur la rue Sherbrooke, en 1847, et l'arrivée de nombreuses postulantes permettent à la communauté de disposer d'un espace et d'un personnel suffisants pour s'occuper d'un plus grand nombre de jeunes filles. En 1847, les religieuses prennent aussi en charge l'éducation de 36 jeunes filles internes, placées au couvent dans une aile à part et dont les parents paient la pension. L'existence de ce groupe de pensionnaires payantes permet de s'assurer un certain revenu et aussi de susciter des vocations parmi les jeunes bourgeoises.

À leur arrivée à Montréal, les religieuses et les personnes dont elles s'occupent consacrent une bonne partie de leur temps à diverses activités manuelles : blanchissage, travaux de couture pour les particuliers et, à partir de 1845, effilage des câbles. ${ }^{(23)}$ Les exercices religieux, le travail manuel et un peu d'instruction constituent alors l'essentiel du programme des religieuses. Comme pour d'autres congrégations masculines et féminines, le travail au Bon-Pasteur n'a pas pour seule fonction d'assurer la survie matérielle, il est aussi présenté comme un moyen d'union à Dieu et un outil efficace pour moraliser et détourner de l'oisiveté. Ce programme ne peut cependant se concrétiser que si les sœurs disposent de moyens suffisants pour assurer la survie de leur œuvre. Tout au long du XIXème siècle et même après, les religieuses du Bon-Pasteur ont à concilier, avec plus
(20) Véronique Strimelle, op. cit., p. 100-101.

(21) Anonyme, Annales des religieuses..., op. cit., p. 26 ; Véronique Strimelle, op. cit., p. 101.

(22) Les anciennes détenues ou les filles repenties qui désirent prendre le voile ne peuvent devenir religieuses du Bon-Pasteur, les Constitutions de la communauté s'y opposent. Pour accueillir ces populations, Mère Marie-de-SainteEuphrasie crée l'institut des madeleines. Les madeleines consacrent leur temps à la prière, au silence et à la solitude afin d'expier leurs "fautes ". Véronique Strimelle, op. cit., 1998, p. 98.

(23) Archives de la Chancellerie de l'archevêché de Montréal, Fonds Sœurs du Bon-Pasteur 
525-107, 844-16, p. 3 ;

Anonyme, Annales des

religieuses..., op. cit., p. $39-40$.

(24) Rapports des inspecteurs de prisons

pour 1869-1870, vol. 4, $\mathrm{n}^{\circ} 12$, documents de la session, p. 39.

(25) Rapports des inspecteurs de prisons pour 1871 , vol. $5, n^{\circ} 1$, documents de la session, $n^{\circ} 31$, p. 6-7.

(26) Anonyme,

Annales des religieuses..., op. cit., p. 165.

(27) Par la suite, en 1895, le gouvernement provincial autorise les Sœurs du Bon-Pasteur à accueillir 45 filles dans chaque école. Archives des Sours du Bon-Pasteur, 236 002-895, contrat du 7 juin 1895 .

(28) Rapport des inspecteurs de prisons pour 1879, vol. $14, \mathrm{n}^{\circ} 1$, documents de la session, $\mathrm{n}^{\circ} 8$, p. 37.

(29) « Acte concernant la procédure dans les causes criminelles ainsi que certaines autres matières relatives à la loi criminelle ", Statuts $d u$

Canada, 32-33 Vict. (1869), ch. 29, art. 79. La loi-cadre régissant les écoles de réforme au Québec se limite, quant à elle, à préciser que les directeurs d'une école certifiée sont tenus de recevoir tous les jeunes délinquants au-dessous de seize ans qui leur seront envoyés, pourvu que leur nombre n'excède pas le chiffre fixé dans le certificat. « Acte concernant les écoles de réforme ", Statuts $d u$ Québec, 32 Vict. (1869), ch. 18 , art. 7 .

(30) En vertu d'amendements apportés à la loi, peuvent aussi être placés en école d'industrie ou de réforme les ou moins de bonheur, ces préoccupations d'ordre spirituel avec leurs besoins pratiques. La diversification progressive de l'œuvre du Bon-Pasteur est à envisager dans cette double logique de conversion et de survie. En recueillant plusieurs types de clientèle, les religieuses peuvent toucher un plus grand nombre de personnes ; en même temps, elles disposent d'une plus large main-d'œuvre et espèrent aussi recevoir de leurs bienfaiteurs et de l'État une aide plus substantielle.

\subsection{L'école de réforme et l'école d'industrie (1869-1912)}

En 1869, le gouvernement du Québec manifeste son désir de créer des écoles de réforme et d'industrie et de les confier à des communautés religieuses. Les Sœurs du Bon-Pasteur proposent alors leurs services en vue de s'occuper des jeunes filles, mais aussi des femmes adultes pour lesquelles le gouvernement provincial voulait établir un nouvel établissement carcéral. Grâce à l'intervention de l'aumônier de la prison de Montréal, l'abbé Huberdeault, du procureur général, Gédéon Ouimet, et de l'inspecteur des prisons, L. Desaulniers, les religieuses obtiennent gain de cause. ${ }^{(24)}$

L'école d'industrie, certifiée le 3 mai 1870, ouvre ses portes le 1er juillet ; l'école de réforme, certifiée elle aussi le 3 mai, ouvre un peu plus tard, le 29 août. ${ }^{(25)} \mathrm{La}$ classe des filles préservées est alors incorporée à l'école d'industrie. ${ }^{(26)} \mathrm{D}$ 'après le contrat passé entre le gouvernement et la communauté du BonPasteur d'Angers, les religieuses peuvent recevoir 45 filles à l'école d'industrie et 30 filles en école de réforme. ${ }^{(27)}$ Pour chaque enfant envoyé à la réforme, 
les religieuses reçoivent 5,50 \$ par mois du gouvernement provincial, alors que la somme allouée mensuellement pour chaque fillette de l'école d'industrie est de $5 \$$. ${ }^{(28)}$ Selon la loi fédérale de 1869 , pouvait être détenu en école de réforme tout enfant de moins de seize ans déclaré coupable d'une infraction pouvant donner lieu à une peine d'emprisonnement. ${ }^{(29)}$ Entre 1889 et 1894, d'autres catégories d'enfants s'ajoutent à ce noyau initial : les enfants placés directement par le secrétaire provincial ; les enfants jugés "incorrigibles", confiés à une école d'industrie, mais dont le comportement (insubordination, évasion) a justifié un nouveau passage devant le juge et un placement dans un environnement plus strict ; les jeunes délinquants en attente de procès et enfin les enfants sourds et muets qui, moyennant certaines conditions, ont quitté les asiles d'aliénés. ${ }^{(30)}$ L'âge minimum requis pour entrer en école de réforme est de sept ans. ${ }^{(31)}$ La durée d'emprisonnement peut aller de six mois à cinq ans.

En ce qui concerne les écoles d'industrie, la loi de 1869 destine ce type d'institution à trois catégories d'enfants. On y trouve tout d'abord les enfants de moins de quatorze ans amenés devant un magistrat parce qu'ils sont abandonnés, errants, fréquentant des voleurs de profession ou dont le père est en prison. La seconde catégorie compte les enfants placés par leurs parents ou tuteurs parce que ces derniers sont incapables de les contrôler. Enfin, le troisième groupe est constitué d'enfants soutenus par une institution et jugés réfractaires. ${ }^{(32)}$ Entre 1869 et 1884, ce sont les enfants de moins de quatorze ans qui peuvent être placés en école d'industrie. La durée d'internement n'est pas précisée et l'âge limite pour entrer en institution ne peut excéder seize ans. ${ }^{(33)}$ Après les amendements de 1884 et 1890, les catégories d'âge admissibles aux écoles d'industrie sont fixées de manière plus limitative : seuls les enfants de sept ans et de moins de douze ans sont admissibles. ${ }^{(34)}$ Avec la loi de 1884, l'âge de sortie obligatoire baisse de seize à douze ans, avant d'être ensuite rétabli à quatorze ans. D'après la loi de 1892, la durée d'internement en école d'industrie peut excéder trois ans et, à partir de 1894, le secrétaire provincial peut décider de prolonger le séjour des enfants en école d'industrie pour une période pouvant aller jusqu'à trois ans. ${ }^{(35)}$ enfants sourds ou muets qui ne peuvent être enfermés en asile d'aliénés parce qu'ils sont considérés comme nonaliénés ou guéris. Cette nouvelle disposition est de courte durée, puisque abrogée en 1892 . Voir Jean Trépanier, Résumé des lois pertinentes aux institutions pour mineurs au Québec, 1846-1915, Montréal, Centre international de criminologie comparée, 1996, p. 26.

(31) Les lois de 1869 ne fixant pas d'âge minimum, il faut se référer aux dispositions du droit criminel précisant qu'un mineur ne peut être tenu responsable criminellement des gestes posés avant son septième anniversaire. Ibid., p. 26.

(32) « Acte concernant les écoles d'industrie ", Statuts du Québec, 32 Vict. (1869), ch. 17, art. 12,14 et 15 . Les lois de 1884 et 1892 viennent 
élargir les conditions

d'admissibilité à d'autres groupes d'enfants, notamment les enfants dont les parents ne peuvent prendre soin à cause de leur maladie, leur pauvreté ou leurs " habitudes vicieuses";

"Acte pour amender

l'acte 32 Vict., ch. 17, concernant les écoles d'industrie ", Statuts $d u$ Québec, 47 Vict. (1884), ch. 23, art. 15. En 1892, la loi destine aussi aux écoles d'industrie les enfants appelés à mourir de faim ou à vagabonder par manque de surveillance parentale ; «Loi concernant les écoles d'industrie ", Statuts du Québec, 55-56 Vict. (1892), ch. 29, art. 2. Enfin, la loi de 1894 élargit à nouveau les populations-cibles en ajoutant les enfants de moins de quatorze ans soumis à des voies de fait, à des assauts indécents, à des outrages quelconques de la part de leurs parents ou

tuteurs ; «Loi amendant les écoles d'industrie ", Statuts du Québec, 57 Vict. (1894), ch. 32, art.

8. Comme nous pouvons le remarquer, les nombreux amendements postérieurs à 1869 ne font que confirmer la lente rupture avec l'ancienne conception de la puissance paternelle, les situations problématiques visées par les lois impliquant de plus en plus la responsabilité parentale. Voir Véronique Strimelle, op. cit., p. 182.

(33) « Acte concernant les écoles d'industrie ", Statuts du Québec, 32 Vict. (1869), ch. 17, art. 15,16 et 36 .

(34) « Acte pour amender l'acte 32 Vict., ch. 17 , concernant les écoles d'industrie ", Statuts du Québec, 47 Vict. (1884), ch. 23, art. 12 et 16.
Ainsi, d'après les classifications opérées par les lois successives, le type de population et les catégories d'âge varient suivant le type d'institution où les filles sont placées. D'après les registres d'écrou tenus par les religieuses, la catégorie d'âge la plus représentée à l'école de réforme durant la période 1869-1912 est celle des treize-seize ans (68 \% du total), tandis que pour l'école d'industrie, ce sont les filles de sept à douze ans qui sont les plus nombreuses $(67 \% \mathrm{du}$ total) ${ }^{(36)}$ La plupart des filles placées en école d'industrie se retrouvaient en institutions par suite du manque de ressources de leurs parents ou tuteurs. ${ }^{(37)} \mathrm{Il}$ existe beaucoup moins de renseignements concernant les motifs justifiant le placement des filles en école de réforme. D'après les bribes, que nous avons pu retrouver, certaines filles sont envoyées "à la réforme" sous le motif de vagabondage, ce qui peut recouvrir une multitude de comportements jugés répréhensibles. En effet, par vagabondage, on peut désigner la simple flânerie ou la prostitution. Dans certains cas, le terme semble aussi employé pour justifier l'enfermement d'adolescentes ayant eu des rapports sexuels hors mariage ou de filles ayant refusé de travailler pour leurs parents. ${ }^{(38)}$

D'après les religieuses, l'implantation des deux écoles au monastère de la rue Sherbrooke entraîne rapidement des problèmes de locaux et de surpopulation, ce qui oblige à transférer le groupe des pensionnaires payantes dans un autre établissement acquis par la communauté. ${ }^{(39)}$ Parallèlement aux écoles de réforme et d'industrie, les religieuses du Bon-Pasteur prirent aussi en charge l'œuvre de la prison des femmes pour lesquelles elles firent construire des locaux sur la rue Fullum. La prison est ouverte en $1876 .^{\left({ }^{(4)}\right.}$ 
Moins de dix ans après l'ouverture des écoles de réforme et d'industrie, en 1878, les religieuses font transférer les filles de l'école d'industrie dans un autre établissement situé à Saint-Hubert, hors de la ville de Montréal. Ce déménagement est le premier d'une longue série de remaniements visant à séparer de façon plus stricte les enfants de l'école de réforme et celles de l'école d'industrie. D'après les religieuses, ces transferts se justifient notamment par le manque de place et par le nombre de plus en plus élevé de filles accueillies en école d'industrie. Outre ces problèmes d'ordre pratique, il semble que la classification entre filles délinquantes et filles en besoin de protection opérée par la loi pose aussi des problèmes. En effet, en 1883, les inspecteurs de prisons constatent que les religieuses maintiennent à l'école de réforme du Bon-Pasteur de Montréal plusieurs petites filles destinées à l'école d'industrie, parce que ces dernières peuvent avoir une influence néfaste sur leurs compagnes. ${ }^{(41)}$ Toujours en vue d'éviter les contacts avec des délinquantes jugées plus endurcies, les religieuses du Bon-Pasteur envoient les filles coupables de délits mineurs à l'école d'industrie :

"Cette école [l'école de réforme] ne reçoit jamais le nombre d'enfants qu'elle a le droit d'avoir parce que la plus grande partie des filles condamnées aux écoles sont presque toutes envoyées à celle d'industrie, n'étant presque toutes nullement coupables de fautes assez graves pour leur mériter une condamnation à l'école de réforme. " ${ }^{(42)}$

Ce classement des clientèles suivant des critères plus moraux que légaux n'est pas particulier au Québec. En Belgique comme en France, les années 1880 marquent aussi un tournant dans la vision de l'enfance. ${ }^{(43)}$ Les discours des administrateurs pénitentiaires et des responsables politiques français et belges insistent en effet sur la nécessité de protéger l'enfance malheureuse et de réprimer avec plus de sévérité les jeunes reconnus incorrigibles. Dans ce contexte où l'enfant apparaît de plus en plus comme une "valeur" à protéger et à rentabiliser car il représente le citoyen de demain, le rôle de la famille et particulièrement des parents fait l'objet d'une attention accrue. L'évolution du regard porté sur l'enfance entraîne à considérer les enfants délinquants et en danger comme des victimes des carences familiales plutôt que comme des acteurs res-
(35) « Loi concernant les écoles d'industrie ", Statuts du Québec, 55-56 Vict. (1892), ch. 29, art. 11 et « Loi concernant les écoles d'industrie ", Statuts du Québec, 57 Vict. (1894), ch. 32 , art. 5 .

(36) Pour une analyse statistique détaillée des populations enfermées en école d'industrie et de réforme au BonPasteur, voir Véronique Strimelle, op. cit., p. 185-

218. Les statistiques ont été élaborées à partir des renseignements tirés des registres d'écrou tenus par la communauté ainsi que des demandes d'internement en école d'industrie faites par la municipalité de Montréal à partir de 1892.

(37) Ibid, p. 206-212.

(38) Au sujet de l'interprétation du terme vagabondage chez les 
filles, voir Tamara

Myers, op. cit., p. 78, et Carolyn Strange, op. cit., p. 254. Les registres d'écrou des Sœurs du Bon-Pasteur n'indiquent les motifs d'internement que pour 78 filles sur 1.028 entrées. Il est évident qu'on ne peut traiter ces données d'une manière approfondie, puisqu'elles n'ont aucune valeur représentative. Véronique Strimelle, op. cit., p. 204-205.

(39) Anonyme, $A u$ soir d'un siècle..., op.cit., p. 56.

En ce qui concerne le surpeuplement des écoles, l'analyse des registres d'écrou montre que les admissions en école de réforme et en école d'industrie ne commencent vraiment à augmenter qu'à partir des années 1880 .

Cependant, il faut aussi noter que les religieuses accueillent dans leurs institutions des filles

placées par charité, qu'elles entretiennent à leurs propres frais. Nous n'en avons trouvé aucune trace dans les registres d'écrou. La surpopulation, dont les religieuses font état, est peut-être aussi liée à une arrivée massive de ce type de détenues. Véronique Strimelle, op. cit., p. 201.

(40) Archives de la

Chancellerie de l'archevêché de Montréal, Fonds

Sœurs du Bon-Pasteur 525-107, 871-1.

(41) Rapport des inspecteurs de prisons pour 1883, vol. 17, documents de la session, $\mathrm{n}^{\circ} 10$, p. 49 . Il faut aussi remarquer que le gouvernement provincial et la municipalité, à la même époque, font également moins de demandes d'internement en école de réforme pour des motifs d'ordre économique, la pension ponsables. Au Québec, ce besoin de séparer les plus jeunes des plus vieux et les innocents des pervertis n'a pas d'impact à court terme. Il faut attendre la loi fédérale de 1908, et son adaptation provinciale de 1912, pour que des changements surviennent dans ce sens dans le domaine de la protection de l'enfance.

Les sœurs continuèrent-elles par la suite à opérer leur propre classement des clientèles ? Nous n'en savons rien. En 1884, l'école d'industrie est transférée à nouveau de l'établissement de Saint-Hubert à la maison-mère. Les religieuses restent cependant préoccupées du besoin de séparer plus radicalement l'école d'industrie et l'école de réforme. Séparation qui se concrétise définitivement en 1895 , lors du transfert de l'école d'industrie dans une nouvelle succursale appelée la maison Notre-Dame de Lorette, située au Nord de Montréal, à Laval-desRapides. ${ }^{(4)}$ Le besoin de déménager dans des locaux plus spacieux se justifiait aussi par la hausse de population au sein de l'école d'industrie, augmentation qui avait débuté, nous l'avons déjà mentionné, au cours des années 1880. En effet, à cette époque, le nombre de plus en plus élevé d'enfants pauvres et laissés à eux-mêmes dans la ville de Montréal entraînait le recours plus fréquent au placement en école d'industrie, ce qui justifiait l'emménagement dans des locaux plus vastes et plus adaptés.

Le déménagement de l'école d'industrie à Lavaldes-Rapides ne soulage que temporairement le couvent de la rue Sherbrooke, puisque les inspecteurs chargés de visiter l'école de réforme font remarquer, en 1900, que le couvent du Bon-Pasteur renferme encore une population bien trop élevée. Il faut dire 
que le nombre d'entrées en école de réforme a connu un regain, qui se maintient jusqu'en 1911. ${ }^{(4)}$ On constate la même tendance à la hausse à l'école d'industrie. Le nombre de plus en plus élevé de demandes d'internement en école d'industrie pour les filles et pour les garçons motive même la création, en 1907, d'un service d'assistance municipale, qui a pour fonction de centraliser et de traiter plus efficacement les demandes d'internement adressées à la ville, afin de mieux discerner les cas de besoin réels. La création de cet organe de sélection ne diminue cependant pas le nombre d'admissions des filles en école d'industrie : entre 1907 et 1912 , le nombre de filles admises à la demande de la ville passe de 58 à 250. ${ }^{(46)}$ Le directeur du service d'assistance n'impute pas cette augmentation à un relâchement de la vigilance de son personnel, mais plutôt à un ensemble de facteurs extérieurs inévitables. Ainsi, en 1908, le nombre plus élevé de demandes serait lié à une grave crise économique affectant le secteur de la construction et plongeant plusieurs familles dans le besoin. La hausse du volume d'internements, remarquable à partir de 1909, s'expliquerait aussi par l'augmentation du nombre d'habitants à Montréal, à la suite de l'intégration par la ville de plusieurs autres municipalités. Outre cette augmentation liée à l'extension territoriale, l'immigration massive de familles pauvres est aussi présentée comme un des facteurs de la hausse des internements. ${ }^{(47)}$ Durant la première décennie du XXème siècle, l'augmentation constante du nombre d'enfants internés dans les écoles d'industrie et de réforme de la ville de Montréal est une des conséquences de l'importance croissante que prend la ville et de l'attraction qu'elle exerce auprès des populations pauvres de la province. Les locaux de l'école d'industrie à Laval ne peuvent alors plus suffire. En 1913, la succursale de Lorette compte 400 fillettes $^{(48)}$ et l'espace manque cruellement. La communauté fait alors construire à proximité de Lorette un nouvel édifice destiné à abriter l'école d'industrie : la maison SainteDomitille, qui ouvre en 1915. Cette nouvelle bâtisse peut notamment abriter de 400 à 500 petites filles. ${ }^{(49)}$ Toujours en 1915 , les jeunes délinquantes de la rue Sherbrooke viennent occuper la maison de Lorette. ${ }^{(50)}$

Le développement des écoles d'industrie et de réforme pour filles à Montréal illustre de façon remarquable l'importance croissante que d'une fille en école de réforme coûtant plus cher aux pouvoirs publics que la pension d'une fille détenue en école d'industrie.

(42) Ibid., p. 85.

(43) Marie-Sylvie

Dupont-Bouchat et Éric Pierre, [dir.], Enfance et justice au XIXème siècle. Essais d'histoire comparée de la protection de l'enfance, 1820-1914. France, Belgique, Pays-Bas, Canada, Paris, PUF, 2001, 443 p.

(44) En 1914, les religieuses font à nouveau construire à Laval-desRapides un autre édifice destiné à l'école d'industrie. La maison SainteDomitille est terminée en 1915, elle accueille l'école d'industrie, tandis que les filles de l'école de réforme quittent Montréal pour occuper la maison de Lorette. Les madeleines sont 
aussi transférées à

Lorette dans une aile à part. Anonyme, $A u$ soir d'un siècle..., op. cit., p. 64.

(45) Véronique

Strimelle, op. cit., p. 197.

(46) Archives de la Ville

de Montréal, v. 001.4/ 1907 à 1912, rapports annuels concernant l'Assistance municipale de 1907 à 1912.

(47) Ibid., p. 3.

(48) Il faut sans doute inclure dans ce chiffre, les filles gardées par charité.

(49) Anonyme, Annales de la maison Sainte-Domitille, auvre de préservation Laval-des-

Rapides, Montréal, Institution des sourdsmuets, 1919, p. 315.

(50) Finalement, une nouvelle maison destinée aux jeunes délin- quantes est construite en 1930, la maison Notre-

Dame de Laval. Ne restent alors sur la rue

Sherbrooke que les

religieuses, les novices et les pénitentes. Une aile du couvent abrite aussi, jusqu'en 1925, le

sanatorium Sainte-

Euphrasie destiné au traitement des femmes alcooliques et morphinomanes.

(51) Anonyme, Règles pratiques à l'usage des religieuses de Notre-Damede-Charité du Bon-Pasteur pour la direction des classes, Angers, Lecoq, 1916, p. 114. prend la communauté dans la gestion des diverses formes de déviance des femmes et des filles au sein de la société du XIXème siècle.

Le placement en école de réforme ou en école d'industrie a pour objectifs de réformer les jeunes délinquantes et d'assurer la protection et l'éducation des petites filles sans tutelle. En examinant les chiffres de plus en plus élevés des populations des deux établissements et en considérant les difficultés matérielles (manque d'espace, de ressources et variations nombreuses des populations) auxquelles les sœurs doivent toujours faire face, on peut se demander si ces institutions atteignent réellement les objectifs qu'elles visent. L'analyse des programmes de traitement que nous allons aborder maintenant pourra peut-être donner des pistes de réponse à cette interrogation.

\section{Le traitement des filles enfermées}

\subsection{Principes éducatifs}

En ce qui concerne l'école de réforme et l'école d'industrie, nous disposons surtout de sources relatives aux discours éducatifs, ce qui ne permet pas de savoir comment ces théories sont réellement appliquées dans les classes. La vie dans les institutions du Bon-Pasteur repose sur un ensemble de règlements et principes tirés des écrits de la fondatrice de la communauté, Mère Marie-de-SainteEuphrasie, celle-ci se révélant très préoccupée par la formation des filles. Dans ses Règles pratiques, publiées une première fois en 1830, la fondatrice du Bon-Pasteur d'Angers ne traite pas uniquement du temps passé en classe. Ses conseils touchent tous les aspects de la vie des enfants en institution. Ils 
apportent donc de précieux renseignements sur le mode de vie que les (52) Ibid., p. 117-118. religieuses entendent imposer aux filles internées. En ce qui concerne l'attitude en classe, Mère Marie-de-Sainte-Euphrasie recommande aux sœurs de toujours manifester beaucoup de bonté envers les jeunes élèves et de se comporter en véritables mères :

«Il faut que tout à la fois vous serviez à vos enfants des classes de guides et de mères. Elles doivent trouver en vous un soulagement dans leurs peines et un remède à leurs maux. " ${ }^{(51)}$

La bonté va de pair avec la miséricorde ; la religieuse doit en effet toujours faire ressortir les éléments positifs présents chez chaque élève :

"Cherchez à voir ce qu'il y a de "bon" dans les enfants qui vous sont confiées. Appliquez-vous à le faire ressortir et en particulier et en public. " ${ }^{(52)}$

À plusieurs reprises, Mère Marie-de-Sainte-Euphrasie insiste aussi sur le fait que la majorité des jeunes filles ne sont pas appelées à la vie religieuse ; il faut donc limiter les exigences à leur égard et les préparer efficacement à gagner leur vie d'une façon honnête en prévision de leur sortie. Même s'il semble dicté par les besoins "réels” de l'enfant, le discours éducatif prôné au Bon-Pasteur obéit néanmoins à une logique de conversion. L'essentiel aux yeux des religieuses est, avant tout, de sauver les âmes des personnes qui leur sont confiées. Certes, il s'agit d'élaborer un ensemble de règles, de pratiques qui pourraient, par leur application systématique, provoquer un "revirement" spirituel chez les élèves. Pour ce faire, il faut créer une ambiance propice à la conversion en organisant les activités des jeunes filles de telle façon que cette transformation se fasse presque à leur insu. ${ }^{(53)}$ Toute fille entrant au Bon-Pasteur se trouve plongée dans un univers clos, où les moindres détails de son existence sont réglés, ordonnés, mais où la réforme morale prime en réalité sur l'instruction. L'adoption d'un mode de vie régulier, la soumission à une stricte discipline sont censés renforcer cette prise en charge de tout l'être en vue d'assurer le succès du programme mis au point au Bon-Pasteur.

Dans le chapitre 16 de ses Règles, tout entier consacré au silence, Mère Marie-de-Sainte-Euphrasie insiste beaucoup sur l'observation scrupuleuse du silence au cours de toute activité. Celui-ci y est présenté comme un 
(54) Ibid., p. 172.

(55) Ibid., p. 134.

(56) Ibid., p. 141.

(57) Ibid., p. 159-160. moyen privilégié de moralisation, car il empêche les "propos coupables et dangereux " et facilite la surveillance. ${ }^{(54)}$ Pour la fondatrice du BonPasteur, la réforme intérieure ne peut réussir qu'au prix de l'isolement. Donc, il faut contrôler et éventuellement supprimer toute source de contacts. En théorie, chaque enfant au travail, au repos ou pendant les loisirs, doit rester surveillée et, idéalement, isolée des autres, les seuls contacts permis et réglementés ne s'effectuant en fait qu'avec les religieuses. On le voit, tout en prônant une attitude plus positive face aux élèves, le discours de Mère Marie-de-Sainte-Euphrasie reste avant tout centré sur le besoin quasi phobique de contrôler les élèves au nom de la prévention. Ce souci reste particulièrement présent dans les écrits touchant à l'application de la discipline au moyen de punitions et de récompenses :

« La punition n'est qu'un remède curatif et souvent impuissant. La grande affaire n'est donc pas de punir, mais de surveiller pour prévoir les fautes, et de les prévenir par la surveillance. ${ }^{(55)}$

En principe, les récompenses doivent être privilégiées pour stimuler les filles et les punitions doivent être utilisées en tout dernier recours. ${ }^{(56)}$ Parmi les récompenses, on trouve les bons points et les bonnes notes. L'entrée dans les "congrégations", sortes de petits groupes d'élite constitués des meilleurs éléments de chaque classe, et l'attribution de "cordons" représentent le summum dans la hiérarchie des récompenses, les congréganistes étant aussi impliquées dans la gestion des classes en tant qu'assistantes de la maîtresse ou que surveillantes.

Dans les Règles pratiques, Mère Marie-de-Sainte-Euphrasie accorde une très grande importance au travail manuel et au travail intellectuel, car ils constituent à ses yeux de puissants instruments de conversion et de formation. Reste à voir maintenant comment ces principes sont mis en pratique au Bon-Pasteur, plus particulièrement pour le travail en ateliers et l'école.

\subsection{Le travail manuel}

En ce qui concerne le travail, la fondatrice du Bon-Pasteur remarque : "Ce serait mal comprendre la vocation des religieuses du Bon-Pasteur que de considérer les classes comme des ateliers de travail et les en- 
fants comme des ouvrières. Pauvres, pour la plupart, ces enfants, sans (58) Ibid., p. 157. doute, doivent gagner leur pain, mais avant tout elles doivent sauver leur âme, puisque c'est uniquement pour ce but qu'elles nous sont confiées. "(57)

Concrètement, même si le travail manuel constitue une source appréciable de revenus pour la communauté, les religieuses responsables des ateliers doivent veiller à ne pas surcharger les enfants de travail et à ne pas prolonger leur temps d'ouvrage. En plus de préparer les enfants à un métier, le travail est considéré comme un moyen d'occuper l'esprit et de préserver de l'oisiveté :

« Il est très important qu'il n'y ait jamais de temps perdu. Quand une enfant, quand une classe surtout n'est pas occupée, il est toujours à craindre que Dieu ne soit offensé. Nous devons donc toujours réfléchir à l'avance et savoir ce que nous ferons faire telle semaine, tel jour, à telle heure, à la classe ou à telle enfant. " ${ }^{(58)}$

Le travail représente aussi un des moyens privilégiés dont la communauté dispose pour assurer l'essentiel de sa subsistance et permettre la survie des multiples œuvres entreprises dans le giron du Bon-Pasteur.

Dans les faits, le revenu du travail des religieuses et des enfants dont elles ont la charge constitue la plus grande part des revenus de la communauté de Montréal. Au monastère du Bon-Pasteur de la rue Sherbrooke et dans les autres succursales créées après 1895, les élèves peuvent travailler dans divers ateliers de couture, de repassage, de blanchissage. Le temps passé en atelier dépend de leur âge, les plus jeunes consacrent l'essentiel de leur temps au travail scolaire, mais plus les élèves sont âgées, plus elles passent du temps en ateliers. ${ }^{(59)}$ En 1888, les sœurs obtiennent de la corporation municipale de Montréal l'autorisation d'établir une buanderie publique dans une nouvelle annexe bâtie pour l'occasion :

«On commença à recevoir du lavage pour les personnes de l'extérieur. Plusieurs enfants de l'école d'industrie aidaient les sœurs à étendre le linge, à le faire sécher [...] Dans la salle à repasser travaillaient cinquante enfants de la réforme. Ces jeunes filles, de dix à vingt ans, sortaient pour la plupart de maisons mal famées. Ce travail aida beaucoup à réformer leur caractère. " ${ }^{(60)}$ 
(61) Archives de la

Chancellerie de l'archevêché de Montréal, Fonds

Sœurs du Bon-Pasteur 525-107, 912-8, règlement destiné aux sœurs directrices (1912).
Cette buanderie publique devient la source principale de revenus des sœurs qui y emploient les filles de l'école d'industrie et de l'école de réforme. La buanderie n'est fermée qu'en 1954.

Entre l'arrivée des sœurs en 1844 et l'établissement des buanderies publiques, le travail des religieuses et des filles devient un élément capital pour la survie du monastère du Bon-Pasteur. Sans les sommes qu'il apporte, l'équilibre budgétaire déjà fragile de la communauté serait gravement affecté. Les initiatives de plus en plus nombreuses des sœurs, à la fin du XIXème siècle, pour élargir et diversifier leur zone d'action obéissent aussi en partie à ce besoin d'accroître leurs sources de financement. En effet, en obtenant entre autres le pouvoir de gérer les écoles de réforme et d'industrie, les religieuses peuvent étendre leur champ d'intervention, bénéficient de subventions gouvernementales et d'un apport supplémentaire de main-d'œuvre à bon marché. Jusqu'à la fin des années 1940, le travail des religieuses et des filles reste dans le programme des activités au Bon-Pasteur. Toutefois, après 1912, le discours sur le travail se transforme, les activités auxquelles les filles se consacrent étant présentées désormais comme des moyens de leur faire acquérir une formation professionnelle. Cependant, vu le profit que les communautés religieuses peuvent tirer du travail des enfants, on peut supposer que, dans certaines circonstances, les activités proposées dans les ateliers s'effectuent surtout pour assurer la survie matérielle des institutions.

\subsection{L'école}

Dans le but de former les filles à une vie " honnête et chrétienne ", les religieuses privilégient dans leur programme les matières pouvant favoriser l'éducation morale des élèves. L'éducation religieuse a donc une place privilégiée dans les matières. La formation ménagère et l'apprentissage des notions élémentaires de lecture, d'écriture, de calcul complètent ce programme visant en principe à rendre les filles capables de gagner leur vie à la sortie de l'institution. ${ }^{(61)}$

Dans les classes, les élèves sont groupées en sections suivant leur âge et leurs aptitudes. Dans la "section enfantine", l'équivalent du jardin d'enfants, les sœurs s'occupent des plus petites âgées de six ans et un 
peu plus. Chez les plus âgées du cours préparatoire, les filles ont quatre heures de cours interrompues par des récréations. Les élèves du cours moyen et supérieur partagent leur journée entre la classe, le travail manuel et les récréations. ${ }^{(62)}$ Les filles de l'école de réforme sont théoriquement tenues séparées les unes des autres dans des locaux distincts. Les fillettes plus petites sont séparées des plus grandes. Pour l'école de réforme, l'horaire d'une journée est sensiblement identique à celui de l'école d'industrie, mais on y compte moins d'heures consacrées à la classe. Les activités du matin se partagent entre les travaux manuels et une demi-heure de catéchisme. Après le repas du midi et une récréation d'une heure, les filles travaillent manuellement durant deux heures et demie, puis suivent deux heures d'enseignement primaire entre 4 et 6 heures. ${ }^{(63)}$ Les dimanches et les jours de congé, les filles peuvent emprunter des livres à la bibliothèque ; elles suivent des cours de politesse.

Il est difficile d'évaluer l'efficacité du régime de gestion de la déviance mis sur pied au Bon-Pasteur et de vérifier si les religieuses ont effectivement réussi leur mission de réforme. Ces dernières disposent de nombreux atouts pour mener à bien leur entreprise : considérées comme des spécialistes dans leur domaine, elles bénéficient du support matériel et moral de personnalités importantes de la société montréalaise, de fonctionnaires influents et, malgré certains conflits, de l'évêque de Montréal. La vie claustrale qu'elles mènent et imposent à leurs élèves facilite la prise en charge totale de ces populations. Cette fermeture au monde leur permet aussi, dans une certaine mesure, d'échapper au contrôle des administrateurs, des inspecteurs et des supérieurs hiérarchiques qui, il est vrai, se préoccupent assez peu des filles. D'un autre côté, le surpeuplement fréquent, le manque de moyens financiers, l'aide limitée du gouvernement provincial et de la municipalité de Montréal, le problème récurrent du manque de place, constituent autant d'entraves au programme initial et ne permettent pas aux religieuses d'agir comme elles le voulaient.

Selon nous, la difficulté à laquelle les sœurs se heurtent dans leur entreprise de réhabilitation, provient aussi de deux volets inconciliables de leur programme : tout en désirant intégrer les filles au monde extérieur en leur apprenant un métier et en les lestant d'un petit bagage de
(62) Les différentes catégories d'âge correspondant aux niveaux scolaires ne sont pas précisées par le règlement ; on peut supposer que les filles du cours supérieur sont celles qui ont douze ans et plus.

(63) Archives des Sours du Bon-Pasteur, 503-020 A, horaire des jeunes personnes condamnées au Bon-Pasteur. 
(64) Archives des Sours du Bon-Pasteur, dossier 701 922, récits de conversion de pénitentes. connaissances, les religieuses présentent comme seul modèle de femme valable celui de la religieuse, de la pénitente, de la madeleine qui quitte le monde pour sauver son âme. Est-ce un hasard si la récompense suprême pour les filles les plus méritantes consiste en une visite chez les madeleines, qui ont pris le voile après être passées en école de réforme ou en école d'industrie ? Les archives du Bon-Pasteur regorgent de récits édifiants de conversions de prisonnières, de jeunes délinquantes passées au Bon-Pasteur. Ces récits suivent toujours à peu près la même trame. Au départ, les jeunes filles sont enfermées contre leur gré. Après une période de révolte plus ou moins longue, un changement intérieur profond suscite leur conversion. Les jeunes filles se repentent de leur passé et, souvent, meurent peu de temps après, apaisées et certaines qu'elles sont "sauvées ". ${ }^{(64)}$ Cette problématique du salut, de la conversion réduit alors les possibilités de réinsertion sociale puisque, implicitement, le seul modèle valable reste celui du retrait du monde. On comprend alors pourquoi les religieuses constatent que certaines filles arrivées au terme de leur internement désirent rester au monastère. Pour certaines, le manque de ressources et les incertitudes de l'avenir ont sans doute motivé leur décision. Ayant passé une grande partie de leur vie dans les murs, nombre de jeunes filles se sont adaptées à la vie institutionnelle et peuvent se sentir inadaptées à l'égard de la vie libre. Pour celles qui ont intégré le modèle présenté par les sœurs, il est quasiment impossible de quitter l'institution sous peine de trahir ce modèle. Dans tous les cas, la jeune délinquante comme la jeune fille pauvre, lorsqu'elles sont sans tutelle, sont implicitement présentées et traitées comme des personnes qui n'ont pas de réelles chances de survie dans le monde. En développant une telle perception de la déviance des filles, les institutions du Bon-Pasteur apparaissent alors plus comme des lieux favorisant le contrôle et la stigmatisation des filles pauvres et délinquantes que comme des écoles de formation morale, intellectuelle et professionnelle.

\section{En guise de conclusion : filles et garçons enfermés, deux mon- des différents}

Jusqu'en 1869 , la volonté de mettre au point un régime de traitement 
spécifique pour les filles est quasiment inexistante. La première loi qui reconnaît le besoin d'un traitement spécial de l'enfance délinquante prévoit l'ouverture d'une prison de réforme destinée en principe aux deux sexes. Cependant, cet établissement ouvert à l'Ile-aux-Noix n'accueille que quelques filles, rapidement jugées indésirables, incorrigibles et renvoyées dans les prisons communes ou dans des pénitenciers à cause de leur "mauvaise influence" auprès des garçons enfermés. ${ }^{(65)}$ Cet épisode est très significatif de la place accordée alors au groupe des filles délinquantes et aux filles en général. La reconnaissance de la délinquance des filles commence en effet à la suite de problèmes essentiellement pratiques liés à une infime portion (trois filles) de la population enfermée à la prison de l'Ile-aux-Noix. À cette occasion, les inspecteurs et le préfet de la prison de réforme évoquent pour la première fois le besoin impératif de séparer les filles des garçons dans des institutions pour mineurs. Pour le Québec, la question de la délinquance des filles n'est donc pas, au départ, perçue comme un problème social, et le besoin de séparer les sexes relève plus du besoin de préserver les garçons des mauvaises influences que de traiter spécifiquement les filles. Les carences et défauts du système d'enfermement des mineurs établi en 1858 justifient le désir de réorganiser et d'élargir les politiques existantes. Réorganiser, en confiant à des communautés religieuses la direction d'institutions d'enfermement spécialisées ; élargir, car ces projets d'institution visent dorénavant les enfants délinquants et les enfants pauvres, abandonnés, vagabonds, ignorés jusqu'alors par les lois.

Dans ce contexte, les lois de 1869 permettent pour la première fois la mise en place d'institutions spécifiques pour les filles et pour les garçons, gérées par des communautés religieuses. La création de ce type d'institutions se révèle alors rentable pour l'Église catholique, puisqu'elle lui permet d'étendre son champ d'influence en matière de gestion du social. Le fait que la plupart des institutions d'enfermement au Québec soient gérées, au XIXème siècle, par des communautés religieuses ne signifie pas pour autant que les types de formation donnée soient tous calqués sur le même modèle. Certes, la plupart de ces communautés ont apparemment beaucoup de points en commun, comme, par exemple, l'importance
(65) Sur les origines de cette première prison de réforme, voir JeanMarie Fecteau, Jean Trépanier, Sylvie Ménard et Véronique Strimelle, op. cit., p. 75-110.

(66) Sylvie Ménard, op. cit., p. 76. 
(67) Rappelons ici que

l'institut Saint-Antoine abrite seulement une école de réforme. Dès l'ouverture de l'institut en 1873 , le gouvernement provincial a passé un contrat avec les Frères de la Charité fixant une pension mensuelle de 13 à $15 \$$ pour chaque élève, deux ou trois fois plus que ce que recevaient les Sœurs du Bon-Pasteur. Véronique Strimelle, op. cit., p. 169.

(68) Sylvie Ménard, op. cit., p. 255.

(69) Ibid., p. 257.

(70) Les Frères de la

Charité ont toujours voulu enseigner des métiers urbains à leurs élèves et, pour cette raison, ont toujours refusé de quitter la ville pour la campagne.

(71) Tout en voulant assurer une formation professionnelle à leurs élèves, les frères tirent aussi profit du travail des garçons qui œuvrent en ateliers pour des manufacturiers, ce qui vaut aux frères de sévères critiques de la part du monde manufacturier qui voit dans le travail des garçons une concurrence déloyale. À ce sujet, voir Sylvie Ménard, op. cit, p. 262-270.

Les métiers disponibles dans les ateliers dépendent aussi beaucoup des disponibilités matérielles (locaux, équipements, argent). Étant donné les problèmes matériels qu'ils doivent affronter au cours du XIXème siècle, les frères ne peuvent cependant offrir autant de métiers qu'ils le veulent et la formation professionnelle des enfants souffre de ce manque de ressources. qu'elles accordent à l'éducation religieuse, ainsi que le rôle moralisateur qu'elles conferent au travail. De grosses différences existent cependant.

À ce sujet, la comparaison entre les Sœurs du BonPasteur et les Frères de la Charité révèle deux "styles" d'intervention fort différents. Grâce à la recherche de Sylvie Ménard portant sur l'institut Saint-Antoine, il est possible de comparer le rôle accordé au travail et à la formation des enfants dans les deux institutions. $\mathrm{Au}$ départ, il faut souligner que, dès l'ouverture de l'institut Saint-Antoine en 1873, la pension mensuelle allouée par le gouvernement provincial pour chaque garçon est de 13 à $15 \$$, soit deux ou trois fois plus que ce que les sœurs reçoivent pour les filles. ${ }^{(66)}$ Même s'ils gèrent une population plus vaste que celle du Bon-Pasteur, les frères bénéficient donc, dès le départ, de moyens financiers plus considérables. ${ }^{(67)}$ Les frères comme les sœurs accordent au travail manuel de grandes vertus, particulièrement celle d'occuper l'esprit et d'éviter ainsi toute forme de désordre. Cependant, le travail à l'institut SaintAntoine revêt une fonction utilitaire beaucoup plus marquée : lors de son ouverture en 1873, l'institut Saint-Antoine est présenté comme une école de métiers industriels. ${ }^{(68)}$ Dans cette perspective, les activités organisées dans les ateliers dépendent des besoins du marché extérieur, ce qui explique en partie les changements des types de métiers pratiqués. ${ }^{(69)}$ À l'opposé, le programme de travail suivi au BonPasteur ne change guère avant les années 1940 et semble cantonner les filles à des tâches plus "traditionnelles" (70) Selon nous, cette différence n'est pas seulement imputable à l'esprit des deux communautés, elle tient aussi au type de population dont ces 
communautés s'occupent et au rôle social que l'on accorde à chaque groupe. Vu le rôle qu'ils sont censés jouer dans la société, les garçons requièrent une formation professionnelle beaucoup plus poussée. D’où le choix des Frères de la Charité, reconnus en Europe pour leurs qualités d'éducateurs et de formateurs auprès des jeunes délinquants. Les besoins et le rôle social des filles sont présentés d'une toute autre façon : ayant une "nature" qui les porte plus à l'intériorisation, les filles sont censées avoir plus besoin de réforme que de formation et doivent essentiellement se consacrer à des tâches ménagères en vue de devenir plus tard de bonnes mères de famille ou de bonnes servantes. D'où le choix d'une communauté religieuse mettant en valeur la réforme intérieure des filles et la formation aux travaux "féminins" : couture, nettoyage, repassage, cuisine...

Contrairement aux religieuses, les frères insistent beaucoup moins sur le salut de l'âme et le besoin d'expiation. Certes, la formation religieuse occupe une place importante dans le programme de l'institut SaintAntoine, mais elle revêt un tout autre sens et poursuit des objectifs différents. Pour les frères, la religion est perçue comme un instrument de moralisation visant à faire des garçons des êtres plus responsables, capables de s'engager dans des activités rentables et socialement acceptables. Pour les religieuses du Bon-Pasteur, la religion a une fonction bien plus "englobante" et plus "intériorisante", l'essentiel de leur programme visant plus, en principe, à convertir qu'à former. Le modèle éducatif appliqué au BonPasteur ne change guère avant les années 1940, alors que les Frères de la Charité administrent l'école de réforme pour garçons en s'adaptant aux besoins changeants du marché du travail. ${ }^{(71)}$

La comparaison entre les modes de traitement des filles et des garçons soulève aussi une interrogation plus fondamentale : tout au long du XIXème siècle, les institutions pour filles du Bon-Pasteur ont suscité beaucoup moins d'intérêt de la part des autorités que l'institut SaintAntoine. On pourrait expliquer cette différence par le fait que les religieuses se montrent en général assez rétives face à toute intervention extérieure, mais cela ne nous semble pas suffisant pour justifier un tel désintérêt. ${ }^{(72)}$ On pourrait aussi expliquer cette situation en arguant du fait que les filles délinquantes ne composent qu'une petite part de la

(72) Les Sœurs du

Bon-Pasteur ont notamment maille à partir avec le représentant de l'évêque, le supérieur ecclésiastique qui se plaint du manque d'ouverture des religieuses et de leur volonté d'indépendance face à l'autorité épiscopale. 
(73) Les filles représentaient environ $10 \%$ de l'ensemble de la population des mineurs détenus.

(74) Véronique Strimelle, op. cit., p. 204-209. population détenue. ${ }^{(73)}$ Cependant, si nous considérons les statistiques des institutions comme des indicateurs du degré de contrôle social plutôt que comme un reflet de la délinquance "réelle" (ce qu'elles ne sont d'ailleurs jamais), on peut se demander pourquoi on enferme moins les filles que les garçons pour des motifs de délinquance. Il ne faut pas oublier en effet que les Sœurs du Bon-Pasteur s'occupent d'une école de réforme et d'une école d'industrie, la première comptant en général moins de filles que la seconde. En fait, l'interprétation accolée au terme "délinquance" differe suivant qu'elle s'adresse aux filles ou aux garçons. Ainsi, quand nous comparons la nature des infractions des filles détenues au Bon-Pasteur et celle des garçons enfermés à l'institut Saint-Antoine, nous constatons que la majorité des délits de garçons sont essentiellement des délits contre la propriété. Nous ne pouvons tirer de conclusions sur les délits ayant conduit les filles en école de réforme du Bon-Pasteur, mais le peu que nous en connaissons laisse à penser que beaucoup de filles sont enfermées à la demande de leurs familles pour des motifs d'ordre moral. ${ }^{(7)}$ Il faut aussi souligner que beaucoup de filles de l'école d'industrie sont enfermées non pour avoir commis des délits, mais pour des motifs de pauvreté ou par suite de mauvais traitements. En ce sens, les filles enfermées au Bon-Pasteur n'ont pas le même profil de déviance, parce que celle-ci est définie suivant d'autres paramètres et d'autres valeurs que celle des garçons. À une époque où le rôle social des femmes s'exerce pour l'essentiel dans la sphère domestique et où le fonctionnement de la société demeure sous la responsabilité exclusive des hommes, il est logique que le problème posé par la délinquance des garçons représente aux yeux des autorités un enjeu politique de plus grande importance, justifiant une attention accrue et la mobilisation de plus amples moyens.

La déviance des filles est plutôt perçue comme une "chute ", une rupture avec le code moral (vagabondage, prostitution, conduite obscène) ou le refus de l'autorité familiale (refus de travailler, oisiveté, désobéissance, rébellion). Faut-il pour autant en conclure que les filles sont "moins délinquantes" que les garçons, comme le prétendent encore certains criminologues? A notre sens, cette double perception est plus une question de regard qu'une question de "nature". 
Sur ce point, il ne faut pas oublier que, délinquantes ou non, les populations détenues en école de réforme et d'industrie appartiennent toutes aux mêmes milieux sociaux et que c'est surtout la pauvreté qui, sous divers motifs, conduit les filles et les garçons en institution. Vue sous cet angle, la mise en place d'institutions spécialisées pour filles et garçons délinquants ou en danger représente alors un épisode crucial du processus de régulation sociale mis en branle par l'État durant le dernier tiers du XIXème siècle. En effet, sous couvert de protection de l'enfance délinquante et malheureuse, l'État peut alors s'immiscer plus aisément dans la vie de familles des milieux populaires qui jusqu'alors restaient hors de son atteinte. ${ }^{(75)}$

(75) À ce sujet, voir Jean-Marie Fecteau, « Note sur les enjeux de la prise en charge de l'enfance délinquante et en danger au XIXème siècle ", Lien social et politiques, 40, automne 1998, p. 129-138. 